Radiology, [online] 39(S1), pp.4-26. Available at: https://link.springer.com/article/ 10.1007/s00247-008-Z1026-3.

4. The Royal Children's Hospital Melbourne (2019). Clinical Practice Guidelines, Radiology - Acute Indications. [online] Available at: https://www.rch.org.au/clinicalguide/guideline_index/Radiology_Guidelines_Acute_indications/.

\section{GP103 STREAMLINING THE PATIENT JOURNEY THROUGH THE COMPLEX NEEDS CLINIC}

\begin{abstract}
${ }^{1,2}$ Niamh Lagan*, ${ }^{3}$ Stephaine Kelly, ${ }^{4}$ Anna Delahunt, ${ }^{3}$ Barbara Fox, ${ }^{3,2}$ Joanne Balfe, ${ }^{3,2}$ Denise Mc Donald. 'Department of Developmental Paediatrics and Neurodisability, Tallaght University Hospital, Dublin, Dublin, Ireland; ${ }^{2}$ Paediatrics, Academic Centre, Tallaght Hospital, Trinity College, the University of Dublin, Dublin, Ireland; ${ }^{3}$ Department of Developmental Paediatrics and Neurodisability, Tallaght University Hospital, Dublin, Ireland; ${ }^{4}$ Department of Nutrition and Dietetics, Tallaght University Hospital, Dublin, Ireland
\end{abstract}

10.1136/archdischild-2019-epa.168

Background The Complex Need Clinic (CNC) in Tallaght University Hospital Dublin, provides a multidisciplinary service to 50 children with exceptional healthcare needs. It is held twice monthly. These children are often non-ambulant, and require enteral feeding. Attending the hospital is often logistically challenging; parents having to organise time off work, transport to and from the hospital, find parking and care for other children, which can lead to stress and anxiety for care givers.

In addition, European Society for Paediatric Gastroenterology, Hepatology and Nutrition (ESPGHAN) 2017 states children receiving enteral nutrition should be reviewed regularly by dietitian and have nutritional bloods annually, and nonambulant children are recommended to have annual hip surveillance as per the Cerebral Palsy Integrated Pathway Scotland (CPIPS) 2013 guidelines.

Aim Increase the number of children attending the $\mathrm{CNC}$ receiving co-ordinated care of non-urgent investigations and synchronised appointments from $48 \%$ to $100 \%$ and improving time efficiency for the patient whilst they attend clinic, by January 2019

Methods Retrospective review of children attending the Complex Needs Clinic revealed only $48 \%$ of children who required hip x-ray monitoring, annual nutritional bloods or dietetic review did not have these completed on the same day. A process map and root cause analysis were completed, involving all stake holders including the parents of children attending the clinic. Opportunities for improvement were identified. Time in motion studies were preformed post intervention.

Results The root analysis and process map identified two areas for improvement; a pre-clinic chart review to highlight patients requiring investigations and pre-ordering of bloods and radiological investigations would stream line the patient journey. Initial time in motion study revealed patients would spend up to greater than $60 \%$ of their time in clinic waiting to be seen. Post implementation of pre-clinic review, co-ordination of same day appointments rose to $100 \%$, and waiting times were reduced to less than $25 \%$ of total time spent in clinic.

Conclusion The implementation of a preclinic chart review clearly identified patients requiring investigations allowing for improvement in adherence to guidelines and improved handover of clinical information. By mapping the process and discussion with families have identified further opportunities for change, better access to disability parking spaces and obtaining a hoist for weighing and changing non ambulant children. A clinical proforma has been developed to assist parents and clinicians increase the efficiency of medical reviews. Ideally, the presence or access to pharmacy support would further enhance the patient journey.

\section{GP104 ARACHNOID CYSTS IN A PAEDIATRIC POPULATION - PRESENTATION AND OUTCOME}

${ }^{1}$ Anda Dumitrescu* ${ }^{*}{ }^{1}$ Michael Moore, ${ }^{2}$ Darach Crimmins, ${ }^{1}$ Niamh McSweeney. ${ }^{1}$ Cork University Hospital, Cork, Ireland; 'Temple Street Children's University Hospital, Dublin, Ireland

\subsection{6/archdischild-2019-epa.169}

Introduction Arachnoid cysts are the most common type of brain cysts, reported in $1 \%$ of the population, with a male predominance. The likely cause is a split of the arachnoid membrane. The majority are thought to be asymptomatic $(80 \%)$ and stable but can present with symptoms of raised intracranial pressure, sometimes secondary to trauma.

Objectives We sought to determine the prevalence of arachnoid cysts in a paediatric population, presenting symptoms, if any, location of cyst, complications, management and outcome.

Methods We did a retrospective review of our radiology database to ascertain cases of arachnoid cysts diagnosed antenatally or postnatally by ultrasound, computed tomography or magnetic resonance imaging in Cork University Hospital over a 3year period (2014-2017) followed by review of their clinical notes. Outcome was classified as improved (cyst reduction in size or complete resolution), unchanged (no clinical worsening, cysts unchanged in size) and worse (clinical deterioration or radiological progression of cyst).

Results There were 62 cases diagnosed in the above time period: $16<1$-year-old (26\%), 29 (47\%) aged 1-10 years and $17(27 \%)>10$ years old. There were 2 antenatally diagnosed, both in the last trimester of pregnancy. There was a male predominance occurring in 58\% (36/62). Indications for neuroimaging included seizures (17), developmental delay (15), headaches (11), head injuries (5) and miscellaneous (14) which included psychiatric symptoms, precocious puberty and neurocutaneous syndromes. One of the neonates underwent endoscopic fenestration of the arachnoid cyst in the second month of life with additional need for cystoperitoneal shunt at 7 months old. Surgery was performed in 6 cases $(10 \%)$, while $56(90 \%)$ were conservatively managed with frequent clinical and imaging monitoring. Seven cases had surgical intervention which included endoscopic fenestration in 5/7 cases $(71 \%)$, craniotomy and evacuation in $2 / 7$ cases (28\%). Two of the 5 cases who had endoscopic fenestration also required shunting. Surgery of symptomatic cases provided overall good results.

Conclusions The majority of arachnoid cysts in our study, remained stable and didn't require surgical intervention. Surgical treatment of intracranial arachnoid cysts remains controversial owing to limited understanding of its natural history and physiology. Current literature favours endoscopic interventions for complicated arachnoid cysts. 